\title{
Programa para mejorar marcadores de riesgo cardiovascular en escolares mexicanos
}

\author{
Otilia Perichart-Perera, MC, RD, (I) Margie Balas-Nakash, LN, ${ }^{(1)}$ Valeria Ortiz-Rodríguez, MC,(I) \\ José Antonio Morán-Zenteno, M en C, ${ }^{(2,3)}$ José Luis Guerrero-Ortiz, MSP, ${ }^{(3)}$ Felipe Vadillo-Ortega, MD.(1,4)
}

\section{Perichart-Perera O, Balas-Nakash M, Ortiz-Rodríguez V, Morán-Zenteno JA, Guerrero-Ortiz JL,Vadillo-Ortega F. Programa para mejorar marcadores de riesgo cardiovascular en escolares mexicanos. Salud Publica Mex 2008;50:218-226.}

\section{Resumen}

Objetivo. Evaluar el efecto de un programa de actividad física sobre los marcadores de riesgo cardiovascular en escolares mexicanos. Material y métodos. Escolares de dos escuelas públicas de Querétaro ( $n=360$, 8-I4 años) realizaron una rutina de actividad física durante 16 semanas (febrero a mayo de 2006). Se compararon mediciones antropométricas, de presión arterial y química sanguínea, antes y después de la intervención. Resultados. La presión sistólica, los triacilglicéridos y el colesterol total disminuyeron de forma significativa en los escolares. La reducción de los lípidos fue mayor en los escolares con valores iniciales alterados. En niñas con riesgo cardiovascular inicial, el puntaje de conglomerado de riesgo disminuyó en grado considerable. No se observaron cambios en el IMC, circunferencia de cintura e insulina sérica. Conclusiones. La aplicación de una rutina de ejercicio sencilla tiene efectos notorios sobre los indicadores de riesgo cardiovascular en escolares. Estos resultados pueden considerarse un modelo de intervención para paliar los efectos de la obesidad infantil.

Palabras clave: niños; actividad física; riesgo cardiovascular; obesidad; México
Perichart-Perera O, Balas-Nakash M,

Ortiz-Rodríguez V, Morán-Zenteno JA,

Guerrero-Ortiz JL, Vadillo-Ortega F.

A program to improve some cardiovascular

risk factors in Mexican school age children.

Salud Publica Mex 2008;50:218-226.

\section{Abstract}

Objective. To assess the effect of a physical activity intervention on cardiovascular risk factors in Mexican school-age children. Material and Methods. Children from two public schools in Queretaro ( $n=360,8$-I 4 years old) performed a 20 -minute physical activity routine every school day during 16 weeks (February-May 2006).Anthropometric, blood pressure and biochemical assessment was done before and after implementation. Results. Systolic blood pressure, triglyceride and total cholesterol levels decreased significantly. The decrease in lipid and lipoprotein levels was higher in children with high baseline levels. In high-risk girls, the cardiovascular risk cluster score decreased significantly. No change in BMI, waist circumference, or insulin was observed. Conclusion. A simple physical activity program modified several cardiovascular risk markers in school-age children. These results may be taken as a reference to develop better intervention programs directed toward preventing the effects of children obesity.

Key words: physical activity; children; cardiovascular risk; obesity; Mexico

(I) Dirección de Investigación, Instituto Nacional de Perinatología Isidro Espinosa de los Reyes. México, DF.

(2) Fundación Actívate, AC.

(3) Secretaría de Salud del Estado de Querétaro, México.

(4) Departamento de Salud, Universidad Iberoamericana, plantel Santa Fe. México DF.

Fecha de recibido: 2 I de agosto de 2007 • Fecha de aceptado: 4 de diciembre de 2007 Solicitud de sobretiros: Otilia Perichart-Perera. Instituto Nacional de Perinatología Isidro Espinosa de los Reyes. Montes Urales 800, col. Lomas Virreyes, I 1000 Miguel Hidalgo, México, Distrito Federal.

Correo electrónico: o.perichart@servidor.inper.edu.mx 
L obesidad infantil es un problema de salud pública en México, cuya prevalencia en escolares ha aumentado de forma alarmante durante los últimos años, desde $18.6 \%$ (1999) hasta 26\% (2006). ${ }^{1}$ Sin embargo, en escolares de zonas urbanas de la Ciudad de México la prevalencia podría ser hasta de $45 \%$, como lo muestran algunos estudios disponibles. ${ }^{2}$

Las dietas hiperenergéticas, los cambios en el consumo de nutrimentos (mayor consumo de grasas y azúcares), el cambio en la dinámica familiar y laboral, el aumento del sedentarismo, entre otros, son factores que han contribuido al incremento general de la obesidad en la población. Algunos estudios enfocados en la población infantil han señalado que el sedentarismo, en relación con ver televisión o usar videojuegos, representa 4.1 horas al día y se ha calculado que cada hora adicional de televisión incrementa el riesgo de ser obeso en 12 por ciento. ${ }^{3}$

Los riesgos vinculados con la obesidad en la infancia incluyen muchas de las comorbilidades que se han descrito en el adulto, entre ellas el conglomerado de factores de riesgo cardiovascular, como hipertensión, hiperinsulinemia, hipertriacilgliceridemia, hiperglucemia y dislipidemia, ligados al síndrome metabólico. ${ }^{4-6}$

Las escuelas se han reconocido como ambientes propicios para promover estilos de vida saludables en los escolares. ${ }^{78}$ Algunos programas de nutrición y actividad física en otros países han resultado efectivos para mejorar hábitos de alimentación, aumentar el consumo de frutas y verduras y reducir el índice de masa corporal (IMC) o la grasa corporal. Sin embargo, no existe ninguna intervención del todo efectiva para prevenir la obesidad o abatir el riesgo cardiovascular en escolares. ${ }^{9}$ A pesar de ello, la actividad física parece jugar un papel central en la reducción del riesgo cardiovascular en escolares y adolescentes. Algunos estudios han demostrado una mejoría en el perfil de lípidos sanguíneos, sobre todo de triacilglicéridos, así como la disminución de la presión arterial al instituir medidas de actividad física en escolares. ${ }^{9-12}$

Dado que es urgente prevenir las consecuencias en la salud derivadas de la obesidad infantil, y en tanto se desarrollan pruebas y esquemas dirigidos a modificar los estilos de vida, es preciso plantear la necesidad de evaluar los efectos sobre marcadores de riesgo cardiovascular de un programa de actividad física aplicado en escuelas urbanas públicas del estado de Querétaro.

\section{Materiales y métodos}

Para este estudio se seleccionaron, por conveniencia, dos escuelas públicas del estado de Querétaro, situadas en la Delegación Centro Histórico, Municipio de Santiago de Querétaro. En total se incluyó a 360 escolares de 8 a 14 años de edad.

La Secretaría de Salud, la Secretaría de Educación Pública del estado de Querétaro y el Comité de Ética del Instituto Nacional de Perinatología Isidro Espinosa de los Reyes aprobaron el proyecto. La organización no gubernamental Fundación Actívate AC diseñó el programa de intervención denominado ACTIVA2 y se instituyó en escuelas urbanas de la ciudad de Querétaro. ACTIVA2 es un programa de actividad física y orientación alimentaria para escolares, diseñado de manera inicial para promover un adecuado equilibrio de energía; su objetivo principal es aumentar la actividad física en los escolares, así como suministrar mensajes que ayudaban a lograr este equilibrio. Se diseñó una rutina de ejercicio de 20 minutos, de acuerdo con los lineamientos del Programa Nacional de Actividad Física (Comisión Nacional de Cultura Física y Deporte). La rutina incluía una fase de calentamiento (3-5 minutos), una fase de flexibilidad, fuerza y balance (5-10 minutos) y una fase de relajación (seis minutos). Esta rutina fue obligatoria para todos los escolares que asistían a las escuelas y era guiada por un educador físico antes del inicio matutino de clases (cinco veces por semana) por 16 semanas. El tiempo de intervención y la duración de la rutina se eligieron con base en estudios previos. ${ }^{11,13}$ Los maestros proporcionaron mensajes de orientación alimentaria y de realización de actividad física a todos los escolares durante sus horas de clase (un mínimo de 30 minutos a la semana) para promover un correcto equilibrio de energía. Dos nutriólogas supervisaron la aplicación del programa mediante visitas diarias a cada escuela.

Se invitó a participar a todos los escolares de las escuelas seleccionadas para realizar una evaluación completa antes de iniciar el programa y después de cuatro meses de su institución. Se obtuvo una carta de consentimiento de los padres y de los escolares que aceptaron participar $(n=360)$. Una nutrióloga clínica realizó todas las mediciones antropométricas y las de presión arterial. La evaluación antropométrica incluyó la medición del peso (Báscula SECA 750), estatura (Estadímetro SECA 206) y circunferencia de la cintura (cinta de fibra de vidrio, SECA 200). Se calculó el índice de masa corporal (IMC) con base en los criterios del Centro de Control de Enfermedades de Estados Unidos de América para su clasificación (curvas CDC). ${ }^{14}$

Las mediciones se realizaron con una técnica estandarizada a partir de los criterios propuestos por Loh$\operatorname{man}^{15}$ (coeficientes de variación $<2 \%$ ), excepto por la medición de la circunferencia de la cintura, en la cual se usaron los lineamientos de los Institutos Nacionales de Salud de Estados Unidos de América. ${ }^{16} \mathrm{El}$ riesgo de 
obesidad abdominal se definió como una circunferencia de cintura $\geq 90^{\circ}$ percentil por edad y sexo. ${ }^{17,18}$

La presión arterial se cuantificó por medio de un esfingomanómetro de mercurio (Tycos CE0050) de acuerdo con la técnica estandarizada y los criterios de la American Heart Association ${ }^{19}$ (coeficiente de variación $<5 \%$ ). Se consideró hipertensión arterial cuando la presión sistólica o diastólica fue $\geq 90^{\circ}$ percentil de acuerdo con la talla/edad y el sexo, con base en el promedio de dos mediciones..$^{20}$

Enfermeras pediátricas tomaron una muestra de sangre venosa en ayuno $(5 \mathrm{ml})$ y se realizó la cuantificación de las concentraciones plasmáticas de glucosa (método de oxidasa de glucosa), albúmina (método de bromocresol), colesterol total (método Trinder), colesterol-HDL (método de magnesio y sulfato de dextrano), colesterol-LDL (fórmula de Friedwald) ${ }^{21}$ y triacilglicéridos (método Trinder) 22 con un analizador automático (Lory 2000) (coeficientes de variación $<2 \%$ ). Las concentraciones de insulina se cuantificaron con pruebas ELISA (DSL.10.1600).

Los valores de riesgo utilizados para las variables bioquímicas fueron: triacilglicéridos altos $\geq 75^{\circ}$ percentil por sexo y edad, colesterol total limítrofe y elevado de $170-199 \mathrm{mg} / \mathrm{dl}$ y $\geq 200 \mathrm{mg} / \mathrm{dl}$, respectivamente; colesterol-LDL limítrofe y elevado de 110-129 mg/ dl y $\geq 130$ $\mathrm{mg} / \mathrm{dl}$, respectivamente; colesterol-HDL bajo como $\leq 25^{\circ}$ percentil por sexo y edad, concentraciones elevadas de glucosa como $\geq 110 \mathrm{mg} / \mathrm{dl}$ e hiperinsulinemia como $\geq 75^{\circ}$ percentil por sexo y edad. 5,7 Para evaluar la resistencia a la insulina se calcularon el índice glucosa/insulina (FGIR) y el modelo de evaluación de homeostasis (HOMA), ${ }^{23,24}$ para lo cual se consideró la presencia de resistencia a la insulina con un FGIR $<7$ y un HOMA $\geq 75^{\circ}$ percentil.

Se recurrió a una estadística descriptiva, pruebas de diferencia de medias (prueba $t$ de Student pareada, Wilcoxon) y la prueba de correlación de Spearman, mediante el programa de cómputo SPSS (versión 13.0). Se analizaron los factores de riesgo de manera individual y se calcularon diferencias por sexo y categorías de edad ( $\leq 9$ años, 10 años, 11 años y $\geq 12$ años de edad). Para analizar el conglomerado de factores de riesgo se cuantificó el puntaje $Z$ de algunas variables (presión sistólica, circunferencia de cintura, colesterol total, colesterol-HDL, triacilglicéridos y HOMA) y se ajustó por sexo y grupo de edad. Las variables que no presentaban distribución normal se transformaron previamente $\left(\log \mathrm{N}_{\text {o }} X^{2}\right)$. Con ello se obtuvo un puntaje del conglomerado de riesgo cardiovascular, tras sumar todas las variables estandarizadas. Se evaluó la diferencia de medias entre los puntajes antes y después de la intervención. Para evaluar las diferencias de acuerdo con el riesgo inicial se consideró que aquellos escolares con un puntaje $>1$ desviación estándar $(D E)$ presentaban riesgo cardiovascular inicial.

\section{Resultados}

De los 360 escolares, 191 (53.1\%) fueron niñas y 169 (46.9\%) niños, con límites de edad de 8-14 años. En la figura 1 se muestra la distribución de los escolares por edad, sexo y presencia o ausencia de obesidad. La prevalencia de sobrepeso fue de $19.4 \%$ y la de obesidad de $22.8 \%$. Se observó obesidad abdominal en $9.2 \%$ de los escolares, hipertensión arterial sistólica en $12.2 \%$ e hiperinsulinemia en 26 por ciento. Ninguno de los escolares mostró hiperglucemia. En cuanto a los lípidos, se identificó hipertriacilgliceridemia en $25.3 \%$ de los escolares, hipercolesterolemia en $13.3 \%$, concentraciones bajas de colesterol-HDL en $25.2 \%$ y concentraciones elevadas de colesterol-LDL en 18.6 por ciento.

El peso, la estatura, el IMC, la circunferencia de la cintura y la presión arterial se incrementaron con la edad, sin mostrar diferencias por sexo. No se reconocieron diferencias significativas en las variables bioquímicas por sexo o grupo de edad, excepto en los triacilglicéridos y la insulina, en los cuales las concentraciones fueron mayores en las niñas.

\section{Intervención}

Hasta 95\% de los escolares completó el programa de actividad física, de acuerdo con la asistencia diaria informada en las escuelas. No se contó con la infraes-

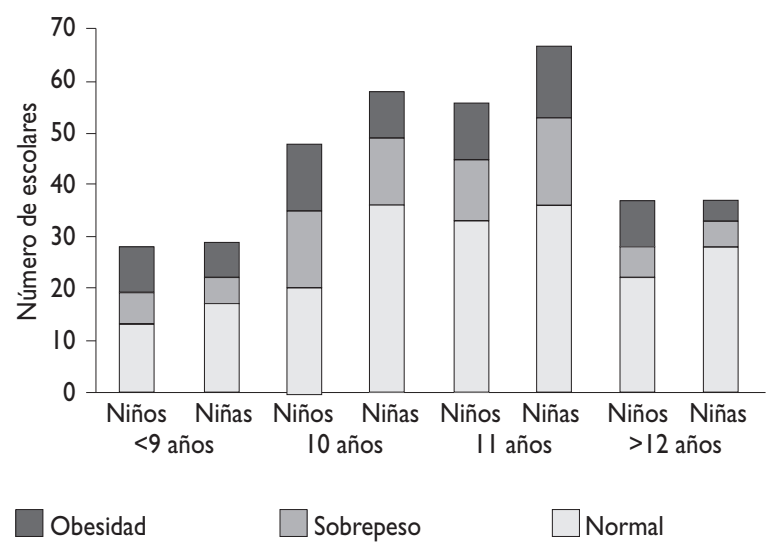

Figura I. Distribución de escolares de acuerdo con la edad, SEXo Y PRESENCIA de obesidad. Querétaro, MéXico, 2006 
tructura para evaluar el efecto específico de los mensajes de orientación alimentaria en el salón de clases sobre los resultados al final de la intervención. Como la estrategia principal del ACTIVA2 fue la rutina de actividad física, se presentan los datos de evaluación de este componente.

Se observó una mejoría significativa de la presión arterial sistólica, colesterol total y triacilglicéridos después de la intervención. La presión arterial sistólica se redujo en $63.9 \%$ de los escolares, con una disminución significativa de $-4.79 \mathrm{mmHg}$ en niños y $-2.97 \mathrm{mmHg}$ en niñas $(p=0.000)$, excepto en el grupo de 10 años. Los triacilglicéridos decrecieron en grado significativo en $62.2 \%$ de los escolares $(p=0.000)$, salvo en niños de 10 años y niñas menores de 9 años. El colesterol total también disminuyó de forma notoria en los escolares $(p=0.000)$ (cuadro I). Los cambios observados en lípidos y lipoproteínas fueron dependientes de las concentraciones iniciales. Se identificaron correlaciones significativas entre las concentraciones iniciales de lípidos y lipoproteínas con los cambios observados (valor inicial - valor final) durante la intervención. Las correlaciones más sólidas se presentaron en los triacilglicéridos y el colesterol-HDL $(r=-0.517$ y $r=-0.465$, respectivamente) (cuadro II).

La disminución de los triacilglicéridos fue mayor en el grupo de escolares que iniciaron el estudio con concentraciones altas $(-56.67 \mathrm{mg} / \mathrm{dl})$, al compararse con aquéllos con valores iniciales normales $(-5.77 \mathrm{mg} / \mathrm{dl}$, $p=0.000$ ). Sólo los niños que iniciaron con valores de triacilglicéridos normales no presentaron cambios sig- nificativos $(-3.75, p=0.349)$. De igual manera, se observó una reducción mayor del colesterol total en escolares con alteraciones al inicio del estudio en comparación con los escolares con valores normales iniciales $(-20.13 \mathrm{mg} / \mathrm{dl}$ contra $-4.42 \mathrm{mg} / \mathrm{dl}$, respectivamente, $p=0.000$ ) (cuadro III). A pesar de la disminución de las concentraciones de colesterol total, de forma paradójica el colesterol-HDL decreció en $79.1 \%$ de los escolares $(p=0.000)$ y el colesterol-LDL aumentó en 67.2\% ( $p=0.000)$ (cuadro I). Estos cambios afectaron de forma selectiva a los escolares que iniciaron el estudio con concentraciones normales de las dos lipoproteínas, en los que disminuyó la concentración de colesterol-HDL $(-7.03 \mathrm{mg} / \mathrm{dl}, p=0.000)$ y aumentó la concentración de colesterol-LDL $(+14.18$ $\mathrm{mg} / \mathrm{dl}, p=0.000$ ) al final del programa. Por otro lado, los escolares con concentraciones alteradas al inicio del estudio no mostraron cambios significativos después de la intervención en ninguna de las dos lipoproteínas (cuadro III).

Se observó una correlación significativa entre la presencia de resistencia a la insulina al inicio (FGIR y HOMA) y el cambio en el colesterol-HDL después de la intervención ( $p=0.002, p=0.048$ ). Se encontró una mayor reducción de las concentraciones de colesterol-HDL en los niños sin resistencia a la insulina (HOMA) antes de la intervención ( $p=0.046)$.

Se observó una reducción de la circunferencia de la cintura en $52.2 \%$ de los escolares, que no fue significativa $(-0.49 \mathrm{~cm}$ en niños, $p=0.102, \mathrm{y}-0.5 \mathrm{~cm}$ en niñas, $p=0.132)$. Tan sólo un niño mostró hiperglucemia después de la

Cuadro I

Cambio en las variables de Riesgo por la intervención de actividad física, Según el género. Querétaro, México, 2006

\begin{tabular}{|c|c|c|c|c|c|c|c|c|c|c|}
\hline \multirow[b]{2}{*}{ Variables } & \multicolumn{5}{|c|}{ Niños } & \multicolumn{5}{|c|}{ Niñas } \\
\hline & $n=$ & $\begin{array}{c}\text { Antes de la } \\
\text { intervención } \\
\text { (Media } \pm D E)\end{array}$ & $\begin{array}{l}\text { Después de la } \\
\text { intervención } \\
\text { (Media } \pm D E)\end{array}$ & $\begin{array}{c}\text { Valor } \\
p\end{array}$ & $\begin{array}{c}\text { Cambio } \\
\text { positivo } \\
\%\end{array}$ & $n=$ & $\begin{array}{l}\text { Antes de la } \\
\text { intervención } \\
\text { (Media } \pm D E)\end{array}$ & $\begin{array}{l}\text { Después de la } \\
\text { intervención } \\
\text { (Media } \pm D E)\end{array}$ & $\begin{array}{c}\text { Valor } \\
p\end{array}$ & $\begin{array}{c}\text { Cambio } \\
\text { positivo } \\
\%\end{array}$ \\
\hline Índice de masa corporal $\left(\mathrm{kg} / \mathrm{m}^{2}\right)^{*}$ & 169 & $20.24 \pm 3.87$ & $20.14 \pm 3.82$ & 0.041 & $28 \%$ & 191 & $20.15 \pm 4.15$ & $20.13 \pm 4.15$ & ns & $24 \%$ \\
\hline Presión arterial sistólica $(\mathrm{mmHg})^{*}$ & 169 & $102.35 \pm 11.91$ & $97.56 \pm 9.88$ & 0.000 & $66.9 \%$ & 191 & $99.54 \pm 11.56$ & $96.57 \pm 10.56$ & 0.000 & $61.3 \%$ \\
\hline Circunferencia abdominal $(\mathrm{cm})^{*}$ & 169 & $71.4| \pm| 2.06$ & $70.92 \pm 10.75$ & ns & $52.7 \%$ & 191 & $70.20 \pm 11.59$ & $69.69 \pm 10.45$ & ns & $51.8 \%$ \\
\hline Glucosa $(\mathrm{mg} / \mathrm{dl})^{\ddagger}$ & 169 & $88.05 \pm 6.45$ & $93.07 \pm 6.63$ & 0.000 & $24.9 \%$ & 191 & $84.49 \pm 6.33$ & $90.65 \pm 6.86$ & 0.000 & $18.3 \%$ \\
\hline Colesterol total $(\mathrm{mg} / \mathrm{dl})^{\ddagger}$ & 169 & $168.70 \pm 28.62$ & $162.66 \pm 26.9 \mid$ & 0.000 & $58 \%$ & 191 & $166.64 \pm 28.72$ & $159.7 \mid \pm 27.76$ & 0.000 & $66.5 \%$ \\
\hline Triacilglicéridos $(\mathrm{mg} / \mathrm{dl})^{\ddagger}$ & 169 & $111.49 \pm 66.84$ & $96.00 \pm 63.71$ & 0.000 & $62.1 \%$ & 191 & $|37.16 \pm 67.5|$ & II $5.73 \pm 54.38$ & 0.000 & $62.3 \%$ \\
\hline Colesterol-HDL $(\mathrm{mg} / \mathrm{dl})^{*}$ & 169 & $45.07 \pm 11.14$ & $40.02 \pm 9.32$ & 0.000 & $22.5 \%$ & 191 & $42.54 \pm \mid 0.61$ & $37.99 \pm 10.42$ & 0.000 & $18 \%$ \\
\hline Colesterol-LDL (mg/dl)* & 169 & $106.77 \pm 26.67$ & $121.28 \pm 32.74$ & 0.000 & $27.8 \%$ & 191 & $106.00 \pm 25.70$ & $115.75 \pm 30.75$ & 0.000 & $36.1 \%$ \\
\hline Insulina $(\mathrm{mlU} / \mathrm{ml})^{*}$ & 164 & $15.55 \pm 8.92$ & $15.96 \pm 6.74$ & ns & $43.5 \%$ & 188 & $17.52 \pm 8.80$ & $1866 \pm 7.46$ & ns & $39.8 \%$ \\
\hline
\end{tabular}


Cuadro II

Correlación de Spearman entre las concentraciones DE LÍPIDOS Y LIPOPROTEÍNAS ANTES DE LA INTERVENCIÓN* Y EL CAMBIO OBSERVADO POR LA INTERVENCIÓN. Querétaro, México, 2006

\begin{tabular}{lcc} 
Lípidos y lipoproteinas $(n=360)$ & Coeficiente de correlación $(r)$ & Valor $p$ \\
Triacilglicéridos & -0.517 & 0.000 \\
\hline Colesterol total & -0.414 & 0.000 \\
\hline Colesterol-LDL & -0.232 & 0.000 \\
\hline Colesterol-HDL & -0.461 & 0.000
\end{tabular}

* Programa de actividad física intervención. El IMC y las concentraciones de insulina no se modificaron de manera notable (cuadro I).

Los niños con sobrepeso y obesidad al inicio tuvieron un mayor puntaje de conglomerado de riesgo en comparación con aquellos niños con IMC normal ( $p=$ 0.000). Este puntaje no decreció en proporción significativa después de la intervención (cuadro IV).

Se registró una disminución notoria intragrupal en el puntaje del conglomerado de riesgo cardiovascular, tanto en los escolares que se clasificaron como de riesgo como en aquellos sin riesgo ( $p=0.015$ y $p=0.043$, respectivamente). Sin embargo, al dividir por sexo, sólo las niñas mantuvieron esta disminución $(p=0.002)$.

Cuadro III

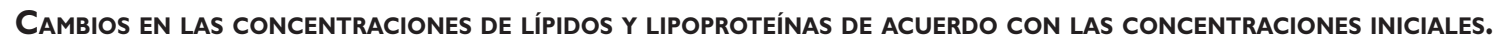
Programa de actividad física. Querétaro, México, 2006

\begin{tabular}{|c|c|c|c|c|c|c|}
\hline Concentraciones iniciales & \multicolumn{2}{|c|}{$n=$} & $\begin{array}{c}\text { Inicial } \\
(\text { Media } \pm D E)\end{array}$ & $\begin{array}{c}\text { Final } \\
\text { (Media } \pm D E)\end{array}$ & $\begin{array}{c}\text { Cambio } \\
\text { (Mediana, IC95\%) }\end{array}$ & Valor $p$ \\
\hline \multicolumn{7}{|l|}{ Triacilglicéridos $(\mathrm{mg} / \mathrm{dl})^{*}$} \\
\hline \multirow[t]{3}{*}{ Elevado } & Todos & 91 & $213.10 \pm 65.76$ & $156.43 \pm 70.57$ & $-62.00(-71.47 a-41.87)$ & 0.000 \\
\hline & Niñas & 48 & $227.10 \pm 57.29$ & $|64.3| \pm 53.25$ & $-57.00(-80.49$ a -45.09$)$ & 0.000 \\
\hline & Niños & 43 & $197.47 \pm 71.54$ & $147.63 \pm 85.72$ & $-69.00(-74.22$ a -25.46$)$ & 0.000 \\
\hline \multirow[t]{3}{*}{ Normal } & Todos & 269 & $95.34 \pm 35.79$ & $89.57 \pm 44.32$ & $-9.00(-11.16$ a -0.38$)$ & 0.037 \\
\hline & Niñas & 143 & $106.97 \pm 36.86$ & $99.43 \pm 44.19$ & $-11.00(-14.99$ a -0.09$)$ & 0.049 \\
\hline & Niños & 126 & $82.14 \pm 29.54$ & $78.39 \pm 41.92$ & $-7.00(-11.57 a+4.07)$ & ns \\
\hline
\end{tabular}

Colesterol total $(\mathrm{mg} / \mathrm{dl})^{*}$

\begin{tabular}{|c|c|c|c|c|c|c|}
\hline \multirow[t]{3}{*}{ Elevado } & Todos & 48 & $214.44 \pm 14.05$ & $194.3 \mid \pm 26.72$ & $-19.50(-26.88 a-13.36)$ & 0.000 \\
\hline & Niñas & 27 & $2|4.26 \pm| 3.4 \mid$ & $193.33 \pm 30.52$ & $-22.00(-31.18 a-10.68)$ & 0.001 \\
\hline & Niños & 21 & $214.67 \pm 15.18$ & $195.57 \pm 21.54$ & $-19.00(-27.43$ a -10.77$)$ & 0.001 \\
\hline \multirow[t]{2}{*}{ Normal } & Todos & 312 & $160.40 \pm 22.96$ & $155.98 \pm 23.66$ & $-5.00(-6.65 a-2.19)$ & 0.000 \\
\hline & Niñas & 164 & $158.80 \pm 22.26$ & $154.18 \pm 23.05$ & $-6.00(-7.64 a-1.60)$ & 0.001 \\
\hline
\end{tabular}

Colesterol-HDL $(\mathrm{mg} / \mathrm{dl})^{\ddagger}$

\begin{tabular}{cccccccc} 
Bajo & Todos & 90 & $31.48 \pm 6.09$ & $32.42 \pm 6.99$ & $-1.00(-1.02$ a +3.02$)$ & $n s$ \\
\hline & Niñas & 50 & $31.48 \pm 5.01$ & $31.31 \pm 7.49$ & $-1.50(-2.7 I$ a $+2.5 I)$ & $n s$ \\
\hline Normal & 40 & $31.49 \pm 7.28$ & $33.80 \pm 6.13$ & $-1.00(-0.8 I$ a +5.55$)$ & $n s$ \\
\hline & Todos & 267 & $47.87 \pm 8.90$ & $41.03 \pm 9.84$ & $-8.00(-7.91$ a -5.75$)$ & 0.000 \\
\hline & Niñas & 138 & $46.55 \pm 9.15$ & $40.18 \pm 10.27$ & $-7.00(-7.76$ a -5.02$)$ & 0.000 \\
\hline
\end{tabular}

Colesterol-LDL $(\mathrm{mg} / \mathrm{dl})^{\ddagger}$

\begin{tabular}{cccccccc} 
Elevado & Todos & 68 & $146.17 \pm 15.15$ & $149.72 \pm 26.27$ & $+0.50(-1.67 \mathrm{a}+9.43)$ & $\mathrm{ns}$ \\
\hline & Niñas & 30 & $148.74 \pm 18.05$ & $150.44 \pm 28.13$ & $+0.50(-7.90 \mathrm{a}+11.04)$ & $\mathrm{ns}$ \\
\hline Niños & 38 & $144.15 \pm 12.27$ & $149.12 \pm 25.00$ & $+0.50(-0.70 \mathrm{a}+12.32)$ & $\mathrm{ns}$ \\
\hline Normal & Todos & 292 & $97.09 \pm 18.24$ & $111.23 \pm 28.46$ & $+10.00(+11.28 \mathrm{a}+16.92)$ & 0.000 \\
\hline & Niñas & 161 & $98.04 \pm 17.86$ & $109.28 \pm 26.68$ & $+6.00(+7.51 \mathrm{a}+14.87)$ & 0.000 \\
\hline & Niños & 131 & $95.92 \pm 18.70$ & $113.62 \pm 30.44$ & $+12.00(+13.40 \mathrm{a}+21.98)$ & 0.000
\end{tabular}

\footnotetext{
* Prueba $t$ pareada

$\ddagger$ Prueba de Wilcoxon ns: no significativo
} 
También se observó un mayor cambio en el grupo de riesgo en comparación con el grupo sin riesgo $(p=0.001)$ (cuadro V).

\section{Discusión}

Los escolares de las dos escuelas públicas de la ciudad de Querétaro analizados en este estudio mostraron una alta prevalencia de sobrepeso y obesidad $(42.2 \%)$ y la presencia de factores del síndrome metabólico en una proporción alta (12\% de hipertensión, 25\% de hipertriacilgliceridemia y $26 \%$ de hiperinsulinemia). Estos datos coinciden con una publicación previa en la cual se demuestra que la obesidad incrementa el riesgo de presentar factores del síndrome metabólico, incluidos hipertensión, hiperinsulinemia, obesidad abdominal e hipertriacilgliceridemia, en escolares de la Ciudad de México. $^{2}$

Esta información permite resaltar la relevancia de iniciar intervenciones dirigidas a paliar los efectos del sobrepeso y la obesidad en la población infantil. Hasta donde se sabe, éste es el primer informe en el que se evalúa el efecto de un programa de actividad física (100 minutos/semana) sobre factores de riesgo cardiometabólicos en escolares de una zona urbana de México. La estrategia de intervención utilizada es sencilla y sus

\section{Cuadro IV}

Cambios en el puntaje del conglomerado de riesgo Cardiovascular de acuerdo con el grado de obesidad. Programa de actividad física. Querétaro, México, 2006

\begin{tabular}{|c|c|c|c|c|c|}
\hline Presencia de obesidad & \multicolumn{2}{|c|}{$n=$} & $\begin{array}{c}\text { Inicial } \\
\text { (Media } \pm D E)\end{array}$ & $\begin{array}{c}\text { Final } \\
(\text { Media } \pm D E)\end{array}$ & Valor $p$ \\
\hline \multicolumn{6}{|l|}{ Conglomerado de riesgo* } \\
\hline \multirow[t]{3}{*}{ Sobrepeso y obesidad } & Todos & 152 & $2.03 \pm 2.98$ & $2.24 \pm 3.10$ & ns \\
\hline & Niñas & 74 & $3.13 \pm 2.6 \mid$ & $3.02 \pm 2.87$ & ns \\
\hline & Niños & 78 & $\mathrm{I} .14 \pm 2.74$ & $|.52 \pm 3.3|$ & ns \\
\hline \multirow[t]{4}{*}{ Normal } & Todos & 208 & $-1.35 \pm 2.58$ & $-1.48 \pm 2.87$ & ns \\
\hline & Niñas & 117 & $-0.62 \pm 2.41$ & $-0.71 \pm 2.38$ & ns \\
\hline & Niños & 91 & $-2.31 \pm 2.65$ & $-2.36 \pm 3.10$ & ns \\
\hline & & & $p=0.000^{\ddagger}$ & $p=0.000^{\ddagger}$ & \\
\hline
\end{tabular}

CuadroV

Cambios en el puntaje del conglomerado de riesgo cardiovascular de acuerdo con el riesgo inicial. Programa de actividad física. Querétaro, México, 2006

\begin{tabular}{|c|c|c|c|c|c|c|}
\hline Concentraciones iniciales & \multicolumn{2}{|c|}{$n=$} & $\begin{array}{c}\text { Inicial } \\
\text { (Media } \pm D E)\end{array}$ & $\begin{array}{c}\text { Final } \\
\text { (Media } \pm D E)\end{array}$ & $\begin{array}{c}\text { Cambio } \\
\text { (Media } \pm D E)\end{array}$ & Valor $p$ \\
\hline \multicolumn{7}{|l|}{ Conglomerado de riesgo* } \\
\hline \multirow[t]{3}{*}{ Riesgo (>I DE) } & Todos & 129 & $3.48 \pm 1.95$ & $2.94 \pm 2.80$ & $-0.54 \pm 2.49 \ddagger$ & 0.015 \\
\hline & Niñas & 83 & $3.66 \pm 1.87$ & $2.80 \pm 2.85$ & $-0.86 \pm 2.50$ & 0.002 \\
\hline & Niños & 46 & $3.17 \pm 2.07$ & $3.20 \pm 2.73$ & $0.20 \pm 2.40$ & ns \\
\hline \multirow[t]{3}{*}{ Sin riesgo $(\leq \mathrm{IDE})$} & Todos & 231 & $-1.78 \pm 2.04$ & $-1.45 \pm 2.78$ & $0.33 \pm 2.48^{\ddagger}$ & 0.043 \\
\hline & Niñas & 108 & $-1.34 \pm 1.81$ & $-0.85 \pm 2.36$ & $0.48 \pm 2.46$ & 0.042 \\
\hline & Niños & 123 & $-2.17 \pm 2.15$ & $-1.98 \pm 3.01$ & $0.19 \pm 2.49$ & ns \\
\hline
\end{tabular}

\footnotetext{
* Prueba $t$ pareada

‡ Diferencia de medias del cambio entre riesgo y sin riesgo ( $t$ de Student)
} 
resultados señalan que se logró modificar el conjunto de factores de riesgo cardiovascular en escolares en un corto periodo de tiempo.

$\mathrm{Al}$ analizar los factores de manera independiente, la presión sistólica decayó en más de la mitad de los participantes, con una disminución clínica considerable de $-4.7 \mathrm{mmHg}$ en niños y $-2.9 \mathrm{mmHg}$ en niñas. En otros estudios de aplicación de ejercicio se han encontrado diferencias similares $(-3$ a $-5 \mathrm{mmHg}) \cdot{ }^{25-27}$ Además, se observó una reducción ligeramente mayor en los triacilglicéridos en los escolares estudiados $(-15.49 \mathrm{mg} / \mathrm{dl}$ en niños y -21.43 mg/dl en niñas) en comparación con lo notificado en otros estudios. ${ }^{10,11,13}$ La diferencia en la disminución de triacilglicéridos y colesterol total de acuerdo con el diagnóstico inicial fue notable. En un análisis de estudios de actividad física sobre factores de riesgo cardiovascular también se notificó una mayor reducción de los triacilglicéridos en los escolares y adolescentes que tenían alto riesgo $(-19.46 \mathrm{mg} / \mathrm{dl}$ contra $-15.92 \mathrm{mg} / \mathrm{dl}) .^{10}$

En contra de lo esperado, otros factores de riesgo cardiovascular no se modificaron de manera favorable con la intervención, incluidos el colesterol-HDL y el colesterol-LDL. Al igual que los triacilglicéridos y el colesterol total, los cambios en las concentraciones de colesterol-HDL y colesterol-LDL fueron dependientes de las concentraciones iniciales. Los cambios negativos en estas dos variables en los escolares con alteraciones iniciales fueron mínimos, respecto del efecto negativo causado en escolares que iniciaron con valores normales. Sin embargo, la variabilidad del cambio fue muy grande en ambos grupos.

Estudios longitudinales que han estudiado el efecto de los programas de ejercicio o entrenamiento sobre las concentraciones de colesterol-HDL y colesterol-LDL en escolares y adolescentes muestran resultados contradictorios. ${ }^{12,28,29}$ En un meta-análisis de los estudios que abordan intervenciones en escolares y adolescentes se muestra que el colesterol-HDL parece modificarse sólo si existen alteraciones antes del programa de ejercicio y que el colesterol-LDL decrece más conforme aumenta la edad y su intensidad. ${ }^{13}$ Las correlaciones entre las concentraciones iniciales de lípidos y lipoproteínas y el cambio en las variables descritas en este estudio son muy similares a las encontradas en un estudio de dosis-respuesta al ejercicio, excepto por el colesterolHDL. ${ }^{12}$ Este efecto dependiente del riesgo inicial se ha informado de forma amplia en la bibliografía, pero aún no se ha propuesto un mecanismo explicativo sólido.

Por otro lado, no se reconocieron cambios en las concentraciones de insulina, si bien la disminución de la concentración de insulina se vincula muchas veces con la pérdida de peso y la reducción de la circunferencia de la cintura. ${ }^{30}$ El periodo de exposición de la intervención evaluada fue corto y ésta no se diseñó para promover la pérdida de peso.

El programa de actividad física fue exitoso al considerar el conjunto de los factores de riesgo cardiovascular, mediante la utilización de un puntaje de conglomerado de riesgo. Los escolares con alto riesgo inicial (>1DE) presentaron una disminución significativa, aunque los escolares sin riesgo mostraron un ligero aumento ( $p=$ 0.043). Debido a la falta de evidencia en cuanto a la relación de actividad física y factores de riesgo cardiovascular individuales, se ha sugerido realizar análisis que agrupen dichos factores de riesgo. ${ }^{31,32}$ En un estudio transversal y multicéntrico, realizado en escolares, en el que se cuantificó de manera objetiva la actividad física, se observó que el puntaje $z$ del conglomerado de riesgo cardiovascular fue menor en aquellos escolares que realizaban la máxima actividad física (último quintil de cuentas por minuto). ${ }^{32}$

La práctica de actividad física se ha reconocido como una de las formas más exitosas para reducir la obesidad y otros factores de enfermedades crónicas no transmisibles. En una revisión sistemática reciente se comunicó que la mayor parte de los programas llevados a cabo en escuelas, en donde la actividad física era la medida principal, tuvo efectos positivos sobre el riesgo crónico. ${ }^{33}$ Las diferencias entre los estudios en cuanto a duración, diseño y metodología hacen difícil concluir aún cuáles son las mejores recomendaciones para reducir el riesgo cardiovascular en escolares obesos y no obesos.

El programa evaluado en este estudio logró abatir tres importantes marcadores de riesgo cardiovascular en sólo cuatro meses de intervención. Sin embargo, el programa de actividad física realizado incluyó menos de 10 minutos de ejercicio aeróbico, el cual se considera el factor esencial para el aumento del colesterol-HDL. ${ }^{34-36}$ Es factible que un mayor volumen de ejercicio podría influir en las variables que no se modificaron. Las tendencias observadas en relación con el colesterol-HDL y LDL permiten pensar que posiblemente con un aumento de la duración, intensidad o frecuencia podría lograrse un cambio positivo en escolares con alteración inicial, sin afectar las concentraciones en los escolares sin alteración inicial.

Existen varias limitaciones reconocidas. Éste es un estudio no controlado, por lo que muchas variables de confusión pueden afectar los resultados. Aunque existe una importante variación diaria en los factores de riesgo descritos, la elevada variabilidad en el cambio de las variables puede atribuirse a la falta de un grupo control y la imposibilidad de controlar las variables de confusión, incluidas la dieta, la actividad física habitual, 
la grasa corporal y la maduración sexual. Se sabe que durante la pubertad se presenta un aumento de la resistencia a la insulina, ${ }^{37}$ así como una disminución de las concentraciones de colesterol-HDL, en relación con los cambios hormonales de esta etapa, y que la actividad física no detiene esta reducción. ${ }^{38,39}$ La actividad física no se cuantificó en los escolares y no es posible asegurar que la actividad física realizada habitualmente modificara los resultados. Sin embargo, no se esperaría que un escolar mejore estos indicadores sin la aplicación de un programa dirigido.

Para compensar la fluctuación de los factores de riesgo se construyó un puntaje de conglomerado de riesgo. Esta alternativa de análisis corroboró que el programa no tuvo efectos en los escolares sin riesgo inicial ni en niños, pero sí se observó un cambio positivo en las niñas que presentaron riesgo al inicio. No obstante, la variabilidad en el cambio del puntaje es aún elevada, lo cual corrobora la presencia de variables de confusión no controladas.

Además, los niños participantes modificaron tal vez los hábitos del estilo de vida por el simple hecho de participar en el programa, lo cual tampoco se evaluó. De igual modo, no se puede descartar que la información suministrada por los maestros en el salón de clases pudo alterar los resultados; empero, este programa no tuvo un componente educativo intenso, el cual es necesario para lograr un cambio de conducta.

A pesar de lo anterior, estos resultados son valiosos, ya que representan un primer esfuerzo que sienta una base para el desarrollo de nuevas medidas, factibles y efectivas. Se logró disminuir la presión arterial sistólica y los triacilglicéridos de manera considerable en los escolares. Se observó que el efecto sobre los lípidos y lipoproteínas fue dependiente de las concentraciones iniciales y que los cambios en los escolares con riesgo inicial son mayores. Las niñas con riesgo cardiovascular inicial, evaluado con factores aislados o con el conglomerado de factores, fueron las más sensibles a presentar cambios positivos. Una estrategia única y sencilla, basada en una rutina de actividad física de 20 minutos por 16 semanas, puede modificar de manera positiva algunos factores de riesgo cardiovascular en escolares mexicanos.

\section{Agradecimientos}

El Programa ACTIVA2 recibió apoyo financiero de la Fundación PepsiCo (EUA) otorgado a la Fundación Actívate y al Instituto Nacional de Perinatología Isidro Espinosa de los Reyes. Los autores agradecen la participación de las siguientes personas que apoyaron el desarrollo técnico y conceptual del proyecto: Isabel
Seoane Jiménez, Concepción Quiroz Téllez, Sofía Tawil Dayán, María Antonia Hernández Miranda, Aurora Espejel Núñez, Araceli Suverza Fernández y Carlos Josafat Castellanos Alejos.

\section{Referencias}

I. Olaiz G, Rivera J, Shamah T, Rojas R, Villalpando S, Hernández M, et al. Encuesta Nacional de Salud y Nutrición 2006. Cuernavaca, México: Instituto Nacional de Salud Pública, 2006.

2. Perichart-Perera O, Balas-Nakash M, Schiffman-Selechnik E, BarbatoDosal A,Vadillo-Ortega F. Obesity increases metabolic syndrome risk factors in school-age children from an urban school in Mexico City. J Am Diet Assoc 2007;107:81-91.

3. Hernandez B, Gortmaker S, Colditz G, Peterson K, Laird N, ParraCabrera S.Association of obesity with physical activity, television programs and other forms of video viewing among children in Mexico City. Int J Obes Relat Metab Disord 1999;23(8):845-855.

4. Freedman D, Dietz W, Srinivasan S, Berenson G. The relation of overweight to cardiovascular risk factors among children and adolescents: The Bogalusa Heart Study. Pediatrics 1999; 103: I 775 - I 82.

5. Weiss R, Dziura J, Burgert T, Tamborlane W,Taksali S, Yeckel C, et al. Obesity and the metabolic syndrome in children and adolescents. N Engl J Med 2004;350(23):2362-2375.

6. Rodriguez-Moran M, Salazar-Vazquez B,Violante R, Guerrero-Romero F. Metabolic syndrome among children and adolescents aged $10-18$ years. Diabetes Care 2004;27(10):2516-2517.

7. Consensus Statement from the American Heart Association. Dietary recommendations for children and adolescents. A guide for practitioners. Circulation 2005; I 12:2061-2075.

8. Bonhauser M, Fernandez G, Puschel K, Yañez F, Montero J, Thompson B, et al. Improving physical fitness and emotional well-being in adolescents of low socioeconomic status in Chile: results of a school based controlled trial. Health Prom Inter 2005;20(2): I I3-I 22.

9. Summerbell C, Waters E, Edmunds LD, Kelly S, Brown T, Campbell K. Interventions for preventing obesity in children (review). The Cochrane Collaboration. USA:Wiley Publishers, 2006.

I0. Froberg K,Andersen L. Mini review: physical activity and fitness and its relations to cardiovascular risk factors in children. Inter J Obes 2005;29:35-39.

II.Tolfrey K, Jones A, Campbell I. The effect of aerobic exercise training on the lipid-lipoprotein profile of children and adolescents. Sports Med 2000;29(2):99-II 2.

12. Tolfrey K, Jones AM, Campbell IG. Lipid-lipoproteins in children: an exercise dose-response study. Med Sci Sports Exerc 2004;36(3):418-427. 13. Kelley G, Kelley K.Aerobic exercise and lipids and lipoproteins in children and adolescents: a meta-analysis of randomized controlled trials. Atherosclerosis 2007;191:447-453.

14. Centro Nacional de Estadísticas de Salud y el Centro Nacional para la Prevención de Enfermedades Crónicas y Promoción de Salud. Tablas de percentiles del Índice de Masa Corporal por edad y sexo para niños y niñas de 2 a 20 años de edad. Estados Unidos, 2000. Disponible en: http://www.cdc.gov/growthcharts/.

15. Lohman T.Advances in body composition assessment. Champaign IL: Human Kinetics Publishers, 1992.

16. National Institutes of Health. National Heart, Lung and Blood Institute. Clinical Guidelines on the Identification, Evaluation and Treatment of Overweight and Obesity in Adults. The Evidence Report. United States, 1998. Disponible en: http://www.nhlbi.nih.gov/guidelines/ obesity/ob_home.htm. 
17. Esmaillzadeh A, Mirmiran P,Azizi F. Clustering of metabolic abnormalities in adolescents with the hypertriglyceridemic waist phenotype.Am J Clin Nutr 2006;83(I):36-46.

18. Maffeis C, Pietrobelli A, Grezzani A, Provera S, Tato L. Waist circumference and cardiovascular risk factors in prepubertal children. Obes Res 200I;9(3): 179-187.

19. Pickering T, Hall J,Appel L, Falkner B, Graves J, Hill M, et al. Recommendations for blood pressure measurement in humans and experimental animals. A statement for professionals from the Subcommittee of Professional and Public Education of the American Heart Association Council on high blood pressure research. Hypertension 2005;45:|42-16I

20. National High Blood Pressure Education Program Working Group on High Blood Pressure in Children and Adolescents. The fourth report on the diagnosis, evaluation, and treatment of high blood pressure in children and adolescents. Pediatrics 2004; I 14(2):555-576.

21 . Frieldwald W, Levy R, Frederickson D. Estimation of the concentration of low density lipoprotein cholesterol in plasma without use of preparative ultracentrifuge. Clin Chem 1972;18:499-502.

22. Trinder P. Determination of glucose in blood using glucose oxidase with an alternative oxygen acceptor.Ann Clin Biochem 1969;6:24. 23. Keskin M, Kurtoglu S, Kendirci M,Atabek M, Yazici C. Homeostasis model assessment is more reliable than the fasting glucose/insulin ratio and quantitative insulin sensitivity check index for assessing insulin resistance among obese children and adolescents. Pediatrics 2005; II5:500-503.

24. Conwell L, Trost S, Brown W, Batch J. Indexes of insulin resistance and secretion in obese children and adolescents. Diabetes Care 2004;27: 3|4-3|9.

25. Bayne-Smith M, Fardy P,Azzollini A, Magel J, Schmitz K,Agin D. Improvements in heart health behaviors and reduction in coronary artery disease risk factors in urban teenaged girls through a school-based intervention: the PATH Program. Am J Public Health 2004;94(9):I538-1543. 26. Hansen H, Froberg K, Hyldebrandt N, Nielsen J.A controlled study of eight months of physical training and reduction of blood pressure in children: the Odense schoolchild study. BMJ 1991;303:682-685.

27. Nader P, Sallis J, Patterson T,Abramson I, Rupp J, Senn K, et al.A family approach to cardiovascular risk reduction: results from the San Diego Family Health Program. Health Educ Q 1989;16(2):229-244.
28. Craig S, Bandini L, Lichtenstein A, Schaefer E, Dietz W.The impact of physical activity on lipids, lipoproteins, and blood pressure in preadolescent girls. Pediatrics 1996;98(3):389-395.

29. Nemet D, Barkan S, Epstein Y, Fiedland O, Kowen G, Eliakim A. Short and long term beneficial effects of a combined dietary behavioral physical activity intervention for the treatment of childhood obesity. Pediatrics 2005; I I5:443-449.

30. Sudi K, Gallistl ,Tröbinger M, Payerl D,Aigner R, Borkenstein M. The effects of changes in body mass and subcutaneous fat on the improvement in metabolic risk factors in obese children after short-term weight loss. Metabolism 200I;50(I I):I323-1329.

3I.Wei C, Srinivasan R, Elkasabany A, Berenson G. Cardiovascular risk factors clustering features of insulin resistance syndrome (syndrome $X$ ) in a biracial (black-white) population of children, adolescents, and young adults: The Bogalusa Heart Study.Am J Epidemiology 1999;I50(7):667-674. 32. Andersen L, Sardinha L, Froberg K, Ekelund U, Brage S, Anderssen S. Physical activity and clustered cardiovascular risk in children: a crosssectional study (The European Youth Heart Study). Lancet 2006;368: 299-304.

33. Flynn M, McNeil D, Maloff B, Mutasingwa D, Wu M, Ford C, et al. Reducing obesity and related chronic disease risk in children and youth: a synthesis of evidence with best practice recommendations. Obesity Rev 2006;7(I):S7-S66.

34. Marrugat J, Elosua R, Covas M, Molina L, Rubies-Prat J.Amount and intensity of physical activity, physical fitness and serum lipids in men.Am J Epidemiol 1996; 143:562-569.

35. Ekelund U, Griffin S,Wareham S. Physical activity and metabolic risk in individuals with a family history of type 2 diabetes. Diabetes Care 2007;30(2):337-342

36. Thompson P, Rader D. Does exercise increase HDL cholesterol in those who need it the most? Arter Thromb Vasc Biol 200 I;21:1097-2000.

37. Goran M, Gower B. Longitudinal study on pubertal insulin resistance. Diabetes 200I;50:2I444-2I450.

38. Eisenmann J. Blood lipids and lipoproteins in child and adolescent athletes. Sports Med 2002;32(5):297-307.

39. Eisenmann J, Malina R.Age-related changes in subcutaneous adipose tissue of adolescent distance runners and association with blood lipoproteins. Ann Hum Biol 2002;29(4):389-397. 